\title{
EVALUATION OF ROTOR STRUCTURAL AND AERODYNAMIC LOADS USING MEASURED BLADE PROPERTIES
}

\author{
Sung N. Jung*, Young-Hyun You \\ Dept. of Aerospace Information Engineering \\ Konkuk University, Seoul, Republic of Korea \\ Benton H. Lau, Wayne Johnson \\ NASA Ames Research Center \\ Moffett Field, California, USA \\ Joon W. Lim \\ Aeroflightdynamics Directorate (AMRDEC) \\ US Army Research, Development, Engineering Command \\ Ames Research Center, Moffett Field, California
}

\begin{abstract}
The structural properties of Higher harmonic Aeroacoustic Rotor Test (HART I) blades have been measured using the original set of blades tested in the wind tunnel in 1994. A comprehensive rotor dynamics analysis is performed to address the effect of the measured blade properties on airloads, blade motions, and structural loads of the rotor. The measurements include bending and torsion stiffness, geometric offsets, and mass and inertia properties of the blade. The measured properties are correlated against the estimated values obtained initially by the manufacturer of the blades. The previously estimated blade properties showed consistently higher stiffnesses, up to $30 \%$ for the flap bending in the blade inboard root section. The measured offset between the center of gravity and the elastic axis is larger by about $5 \%$ chord length, as compared with the estimated value. The comprehensive rotor dynamics analysis was carried out using the measured blade property set for HART I rotor with and without HHC (Higher Harmonic Control) pitch inputs. A significant improvement on blade motions and structural loads is obtained with the measured blade properties.
\end{abstract}

\section{INTRODUCTION}

In 1994, the Higher-harmonic-control Aeroacoustic Rotor Test (HART I) was conducted in an open-jet anechoic test chamber of the German-Dutch wind tunnel (DNW) by a joint research team from the German DLR, the French ONERA, NASA Langley, DNW, and the U.S. Army [1]. The main focus of the task was to measure the noise level, airloads, vortex wake, and blade motions of a rotor in various flight conditions, with and without higher harmonic control (HHC) pitch inputs, with objectives to improve the physical understanding of the blade-vortex interaction (BVI) phenomena and to promote the $\mathrm{HHC}$ techniques for active rotor control. The test data included extensive acoustic and vortex flow measurements which resulted in one of milestone accomplishments in the rotorcraft aeromechanics fields [2].

The remarkable success of HART I brought worldwide attention and led to a succeeding program HART II which was conducted in 2001 in the DNW wind tunnel [3]. Despite the fact that HART I test was completed much earlier than HART II and valuable sources of high precision data were provided, the published efforts associated with HART I are significantly less in volume, as compared with HART II [4]. The representative ones of HART I are Tung et al. [5], Beaumier and Spiegel [6], Brooks et al. [7], Lim et al. [8-9], and Yeo and Johnson [10-11]. Most of the research focused on correlating the airloads and vortex wakes against the measured data. Only a limited attempt has been made to date to validate the structural loads of HART I rotor [11]. To reach an acceptable structural load correlation, a reliable set of blade structural properties is needed.

The HART I rotor is a four-bladed, Mach-scaled model of the BO-105 hingeless main rotor. The blades were composed of E-glass spar and honeycomb materials and were manufactured by Dynamic Engineering, Inc. (DEI) in the early 1990's. Even though some of the measured properties such as the non-rotating blade frequencies are available, the actual blade properties were never measured as a complete set. Previous studies on the validation of HART I rotor [10-11] indicate that the predicted airloads are reasonably matched with the measured data, whereas the structural loads results exhibit poor correlation with the wind tunnel test data, in 
terms of both magnitudes and phases. This suggests uncertainty in the DEI blade property set and there is a need to supply reliable HART I blade properties data which can only be achieved by a careful measurement process. Furthermore, it has been known that up to four different sets of blade property data for HART I are in use [5-11] and there is a clear need for unified version of the blade properties.

In an attempt to resolve the uncertainty issue associated with the HART I blade properties and to provide the required data set to the rotorcraft community, the original blades adopted in the wind tunnel test in 1994 were delivered to the NASA Ames Research Center in 2005 from the German DLR (German Aerospace Center). Since then, a series of laboratory tests have been conducted to experimentally determine the structural properties of HART I blades. The measurements include geometric offsets, bending and torsion stiffness, mass, and inertia properties. In order to ensure the reliability of the measurement data, multiple testing techniques were introduced for specific blade properties. These test techniques include the mirror method [12] and 3-point bending [13] for stiffness properties as well as the trifillar [14] and pendulum methods [15] for inertia properties.

The goal of the present study is to conduct the measurement on structural properties of HART I blades and to generate a unified version of reliable set of measurement data. Given the measured blade property set, a comprehensive dynamics evaluation using CAMRAD II [16] is performed to validate and illustrate the sensitivity of airloads and structural loads of HART I rotor. Special focus is given on whether the newly measured blade properties would improve the structural loads predictions, relative to those observed by Yeo and Johnson [11] where estimated blade property data were used and relatively poor correlations with test data were obtained. In order to address the impact of using the measured structural properties on the validation of HART I wind tunnel test data, the present approach is constructed based upon the comprehensive model used by Yeo and Johnson [11]. The quality of blade structural measurements is evaluated by correlating the comprehensive dynamics predictions against the HART I data, with and without higher harmonic pitch inputs. The present study is believed to be the first attempt to investigate the structural loads correlation for HART I rotor with HHC pitch inputs.

\section{HART I TEST}

The HART I test conditions considered are in descent flight with an advance ratio $\mu=0.15$, a shaft tilt angle $\alpha_{s}=5.3 \mathrm{deg}$ aft $(4.5 \mathrm{deg}$ after the wind tunnel wall and fuselage correction), a hover tip Mach number $\mathrm{M}=0.641$, and nominal thrust level $\mathrm{C}_{\mathrm{T}}$
$=0.0044$. Three test cases are selected for comparison. The first one is the baseline (BL; Run 140) without HHC. The others are the minimum noise (MN; Run 138) which produces lower noise with 3-per-revolution $\mathrm{HHC}$ input of 0.87 deg amplitude and 296 deg phase and the minimum vibration (MV; Run 133) leading to a lower vibration (0.83 deg amplitude and 178 deg phase). More detailed information is available in Ref. 17.

The rotor was a four-bladed, 40\% Mach-scaled hingeless BO-105 model with $2 \mathrm{~m}$ radius and 0.121 $\mathrm{m}$ chord length. The reference blade (blade 1) was heavily equipped with pressure transducers and strain guages distributed along the span and chord. These sensors in blade 1 led to about $6 \%$ heavier weight than the other blades. Almost all the pressure sensors were installed at three radial stations $(r / R=$ $0.75,0.87,0.97)$ to obtain the complete pressure distribution along the chordwise direction. A total of 32 strain guages, spanning between $r / R=0.14$ and 0.83 , were attached on the blade surface to measure the structural loads and the elastic deflections of the blade. An alternative measurement technique by an optical method was also performed to validate the blade elastic deflections. The pressure and strain gauge signals were sampled at a rate of 2,048 per revolution. The experimental test data were averaged over 64 revolutions for each test condition.

\section{BLADE PROPERTY MEASUREMENT}

The instrumented blade (blade 1) would be used to measure the blade structural properties since most of the wind tunnel measurements were carried out with the blade 1 . However, blade 1 was severely damaged during a separate test after HART I and turned out to be inappropriate for further structural test. Instead, measurements were made using the remaining blades. One of the HART I blades (blade 3) was chopped into several pieces to obtain the mass and inertia properties. It is noted that the airfoil section of HART I blade with constant chord starts at $22 \%$ radial station $(0.22 \mathrm{R})$ up to the tip of the blade. For convenience, the inboard of $0.22 \mathrm{R}$ is called as the blade root section and the outboard portion as the blade uniform section. This section briefly describes specific testing methods and procedures for the measurement of HART I blade properties.

\subsection{Section stiffness}

The stiffness measurements are based on two approaches: the mirror method [12] and the 3-point bending technique [13]. The first one uses a pair of mirrors bonded on the blade surface while a sheet of laser light is beamed onto the mirrors. Figure 1 shows the schematic of the mirror method for a fixed-free blade in bending. The laser light is reflected from the mirrors onto the wall separated at a distance $D$. When the load is applied, then the laser reflections on the wall will traverse to a distant 
location $d$. By recording the load applied to the blade and the deflection of laser reflections on the wall, the effective blade stiffness in bending is obtained between each mirror pair as:

$$
E I=\frac{P}{2 \beta}\left(a_{1}^{2}-a_{2}^{2}\right)
$$

where $P$ is the applied force, $\beta(=d / 2 D)$ is the laser path angle, and $a_{1}$ and $a_{2}$ denote the distances between the mirrors and the position of load (see Fig. 1), respectively. Figure 2 shows the actual measurement scene for the flap bending via the mirror method. The torsion stiffness is obtained in a similar manner using the mirror method.

For the 3-point bending, the blade is placed on roller supports to simulate a simply-supported boundary condition while the load is applied at the center. The bending stiffness is then evaluated using the relation:

$$
E I=\frac{l^{3}}{48 \cdot\left(\delta_{c} / P\right)}
$$

where $l$ is the length between the end supports, $\delta_{c}$ denotes the deflection at the center, and $\delta_{c} / P$ represents the slope of the deflection-load curve, respectively. The deflection is measured using a pair of dial gauges having a resolution of 0.001 in each. The slope of the deflection-load curve is determined by averaging over ten different measurements. Figure 3 shows the measurement setup for the chord bending stiffness using the 3point bending method. To this end, special fixtures and loading blocks have been built for the boundary supports and the application of the load, respectively. To ensure the application of the loading being perpendicular to the beam axis of pretwisted HART I blades, a metal plate that can be adjusted to arbitrary angles is placed underneath of each of the supports. Uniformity of blade properties between the end supports is assumed to obtain the elastic property. This rather simple technique has been proved efficient and used extensively for the flap bending and the chord bending of the blade uniform section.

Figure 4 shows the comparison of the measured flap bending, chord bending, and torsion rigidity of HART I blades denoted as a function of the blade span coordinates, against the DEl data. The present measured values are expressed as the continuous lines and the DEI predictions are expressed as the dotted lines. The DEI values are chosen for comparison among the four different set of existing HART I blade properties since the others should be based on the DEl estimations. For the flap bending, both the blade root and the uniform sections are measured while it is measured only at the uniform section for chord bending or torsion. The DEI data are used where the measurement records are not available. It is observed that the DEI values generally overestimate the blade section rigidities by up to $30 \%$ in the flap bending of the root section and $14.3 \%$ in the chord bending of the uniform section. It should be mentioned that all measured values are averaged over 10 different trials with the same setup.

\subsection{Mass properties}

One of the HART I blades (blade 3 ) has been cut into six small pieces to measure the mass and inertia properties of the blade uniform section. The region of the cut sections measures about $1 \mathrm{~m}$ long starting from the blade tip. The weight of each blade section segment has been measured using a mechanical balance. Figure 5 shows the distribution of mass along the blade span obtained using the present measurement against the DEI data. The agreement between the measured data and the DEI data appears good, including the blade tip region where the balancing weight is placed. The chordwise center of gravity (CG) is measured by placing the blade section segments on a knife edge, as demonstrated in Figure 6 . The measurements indicate some scatter between the different sections due to imperfections in the blade cuts. It is finally determined that the measured $C G$ is positioned at $26.5 \%$ chord $(0.265 c)$ from the leading edge of the blade section.

The polar mass moment of inertia (MOI) is measured using a trifilar pendulum method [14]. In this method, a circular disk with known weight and inertia is suspended by three thin cables having equal lengths (see Figure 7). The polar $\mathrm{MOI} I_{\theta}$ is obtained using the relationship given by [14]:

$$
I_{\theta}=\frac{1}{b} \frac{W r^{2} t^{2}}{4 \pi^{2} l_{c}}
$$

where $W$ is the weight of the suspended object, $r$ is the radial distance of the cable attachment from the disk center, $t$ is the time period, $l_{c}$ is the length of the cable, and $b$ is the length of the blade segment, respectively. The measurement is performed in the following sequence: (1) Place the blade uniform section on the suspended disk; (2) Start a pendulum motion with a small initial angle; (3) Obtain the period of oscillations after sufficient number of cycles are proceeded; (4) Repeat the above procedures for an averaged response. Figure 7 shows a photograph taken for the measurement of the polar $\mathrm{MOI}$ for a blade uniform section. A laser sensor as well as the frequency counter (HP 5334B) are used for the period of oscillations. Figure 8 shows the comparison of the polar mass $\mathrm{MOI}$ about the $\mathrm{CG}$ obtained between the present measured values and the DEI data. Since the measurement is based at the 
quarter chord, the measured $\mathrm{MOI}$ values are transferred by applying the parallel axis theorem [18]. It is indicated that the DEI values overestimate the measured $\mathrm{MOI}$ for the blade uniform section by about $9.5 \%$.

\subsection{Elastic axis}

The elastic axis is defined as the chordwise position of the blade where the vertical bending induces no tosional motion. Figure 9 shows the measurement setup for the determination of the elastic axis. A fixed-free blade, having a length of about $946 \mathrm{~mm}$, with a loading fixture at the tip is constructed for this purpose. The position of loading along the chord is varied using a sliding table attached to the loading fixture and the amount of loading is measured by an interface load-cell. To measure the angular displacements, both the dial gauges and the autocollimator are installed near the root and the tip, respectively. Two optical mirrors are mounted on the blade leading edge near the blade tip and they are aligned with the auto-collimator, as indicated in Figure 9. Once the measurement setup is completed, the load is applied first at the quarter chord position and the angle of twist is determined by reading the collimator angle as well as the dial gauges. Next, the chordwise position of loading is moved toward leading-edge or trailing-edge until the relative twist angle becomes nearly zero, which corresponds to the elastic axis position. It is found that the measured elastic axis of the blade uniform section is positioned at $20.5 \mathrm{~mm}(0.169 c)$ from the leading-edge. It is noted that the DEl estimations predicted at $24.2 \mathrm{~mm}(0.2 \mathrm{c})$.

Table 1 summarizes all the measured blade properties records obtained for the uniform section, and the DEI values for comparison.

\section{COMPREHENSIVE ROTOR ANALYSIS}

\subsection{CAMRAD II modeling}

The aeroelastic response of HART I rotor in the wind tunnel is computed using the comprehensive rotorcraft dynamics analysis code, CAMRAD II [16]. The blade structure is discretized into 15 nonlinear beam finite elements. Each beam segment is represented by three translational (axial, flap, and lead-lag) and three corresponding rotational degrees of freedom (DOF), resulting in a fifteen DOF beam finite element. The aerodynamic model used is based on the ONERA-EDLIN unsteady airfoil theory combined with C81 airfoil table look-up. For the vortex wake representation, the free wake geometry is used to compute the non-uniform induced inflow around the rotor. The formation of the tip vortices is modeled using the multiple-trailer wake with consolidation model. A total of 17 aerodynamic panels distributed more densely near the blade tip are used to predict the aerodynamic loads acting on the blade. The multiple-trailer wake model has discrete vortex filaments convected from the aerodynamic panel edges. The trailed filaments are eventually consolidated into a single rolled-up state after a given instant of time by entrainment or compression process [16].

The comprehensive rotor dynamics analysis is carried out for the three test cases of HART I rotor. These include BL, MN, and MV cases. Only an isolated rotor configuration is considered for the present investigation. The effect of a fuselage is taken into account indirectly by correcting the shaft tilt angles by about $0.2 \mathrm{deg}$. The rotor trim is met by matching the measured rotor thrust and hub roll and pitch moments in the wind tunnel condition.

\subsection{Rotor natural frequencies}

The predicted natural frequencies of HART I rotor using the measured structural properties are compared in Figure 10 against the measured data [17], denoted as black circles, as well as the predictions by Yeo and Johnson [11]. The frequencies are non-dimensionalized by the rotor nominal speed ( $\Omega=1040$ RPM). It is noted that the measured frequencies were obtained for the instrumented blade (blade 1) and only available at non-rotating condition. The published results by Yeo and Johnson [11], denoted in Figure 10 as dotted green line and labeled Yeo and Johnson with mod DEI data, were obtained after adjusting the blade stiffnesses and inertia properties from the DEI values to match the measured frequencies. The additional one labeled as Yeo and Johnson with DEI data was reproduced using the original input of CAMRAD II without changing the structural properties given by DEl. Both sets of calculation used a linear pitch link model with the spring constant of 1.23 million $\mathrm{N} / \mathrm{m}$. The present results are denoted as the blue continuous lines and obtained using the measured structural properties without resorting to any kind of tuning. A pitch link stiffness of 0.43 million $\mathrm{N} / \mathrm{m}$ is adopted, which is equivalent to a pitch bearing stiffness of $1706 \mathrm{~N}-\mathrm{m} / \mathrm{rad}$, to match the first torsion frequency of the instrumented blade.

It is observed from Figure 10 that the DEI data result in higher bending and torsion frequencies than the measured frequency values. An artificial reduction in frequencies was introduced in Yeo and Johnson [11] by adjusting the bending and torsion stiffnesses by about $10 \%$. It is seen that the calculated frequencies by the present approach indicate slightly higher frequencies in flap bending. This is mainly due to the fact that the measured frequencies are taken from the instrumented blade while the present structural properties are obtained based on the rest of the blades (i.e., uninstrumented blade). Considering the fact that the instrumented blade is heavier than the other blades by about $6 \%$, the present frequencies appear to have a reasonable correlation with the measured values. 


\subsection{Trim and blade motions}

The trim process matches the measured thrust, roll, and pitch moments specified at the wind tunnel test. Table 2 shows the trim targets given for each of the three test cases of HART I rotor: BL, MN, and MV case. It is defined as positive when the advancing side goes up (roll moment) and the pitch-up motion occurs. A Newton-Raphson iterative scheme with Jacobian evaluations is adopted in CAMRAD II to reach the desired trim. The resulting outcomes from the trim equations are the collective pitch at $75 \%$ radial station $(r / R=0.75)$, the lateral cyclic pitch, and the longitudinal cyclic pitch angles. Table 3 shows the comparison between the present predictions and the measured trim values. The correlation is reasonable for the lateral cyclic pitch and less satisfactory for the collective pitch and the longitudinal cyclic pitch angles. The deviation is generally larger for MV case.

The rigid pitch angles alone does not represent the overall trim behavior, especially when the elastic blade motions play substantial roles [19]. Figure 11 shows the time domain response of the elastic flap displacements at the blade tip, obtained between the present predictions and the wind tunnel measurements for $\mathrm{BL}, \mathrm{MN}, \mathrm{MV}$ cases, respectively. Results by Lim et al. [9] are presented for comparison. As is seen, the present predictions generally underestimate the peak-to-peak values against the measured data. However, the waveforms of the blade response are reasonably predicted with correct phase angles. The underestimation of the blade response is expected since the present predictions are based from the unintrumented blade which is lighter than the instrumented blade. It is noted that the heavier blades exhibit higher peak-topeak deflections for the case with HART II blades [20], which have similar dynamic characteristics with HART I blades. For MN and MV cases, the predicted results by Lim et al. [9] show locally good agreements with the measured data, but the waveforms are biased to the advancing side (MN) or to the retreating side (MV), resulting in different waveforms with the measured blade responses. It should be mentioned that Lim et al. [9] used slightly different set of structural properties where the DEI data were used for the blade uniform section while HART II blade properties were adopted in the root section.

The correlation of the present elastic twist predictions, against the measured data as well as predicted results by Lim et al. [9], is presented in Figure 12 for the three test cases, respectively. The present predictions show good agreements with the measured data in terms of both magnitudes and phases, with a slight under-estimation of the peak-topeak values for MN and MV cases. The 2-perrevolution behavior of the $\mathrm{BL}$ case as well as the 3per-revolution behavior of the HHC input cases are captured nicely with the present approach. In general, the present results demonstrate clearly improved correlation compared to the predictions by Lim et al. [9]. The mean values and the peak-to-peak magnitudes predicted by the present approach are increased significantly, leading to improved prediction against the measured data. It is remarked that the improvement is achieved by the measured blade structural properties in the present comprehensive analysis.

\subsection{Effect of blade structural properties}

The influence of measured structural properties on airloads and structural loads of HART I rotor is addressed in this section. Only the $\mathrm{BL}$ case is considered since the corresponding results by $\mathrm{YeO}$ and Johnson [11] are available for this specific case. The remaining cases with $\mathrm{HHC}$ inputs will be discussed in the separate sections. Figure 13 shows the comparison of section normal forces $M^{2} C_{n}$, computed at three radial stations $(r / R=0.75,0.87$, 0.97 ), where $M$ denotes the local Mach number. In this plot, the present predictions are compared with the measured data and the predicted results by Yeo and Johnson obtained using the DEI data with or without tuning the blade properties. It is noted that results labeled Yeo \& Johnson with DEl data are reproduced using the input deck provided by the authors while results labeled Yeo \& Johnson with mod DEI data [10], obtained at $87 \%$ radial station, are taken from Ref. 10. All predictions used identical aerodynamic models except that a smaller time step size ( $2 \mathrm{deg}$ ) is employed in the post-trim stage of the present analysis, while each Yeo and Johnson's used a $15 \mathrm{deg}$ resolution. The refinement in the time step size affects mostly the high-frequency response such as BVI oscillations and no significant influence is observed on the low-frequency airloads signals.

It is seen that the present predictions capture reasonably the general trend of both low frequency loadings and high frequency BVI events in the measured signals of the three test cases. The change of structural properties is seen to have a non-negligible effect on section airloads. Specifically, the peak-to-peak values of the predicted $M^{2} C_{n}$ with the DEI data become increased substantially as compared with the present predictions and the modified DEI properties adopted in Ref. 10. This is because the blades with DEI data are relatively stiff and softening of the blade properties by the tuning process results in higher aerodynamic loadings.

The effect of measured blade properties on oscillatory structural loads components for BL case is presented in Figure 14. The comparison is again made against the measured data and $\mathrm{Yeo}$ and Johnson with and without tuning the DEI data. Similar to the previous case, results labeled Yeo \& Johnson with DEI data are reproduced using the original input deck while results labeled Yeo \& Johnson with mod DEI data [10] are obtained from 
Ref. 11. The oscillatory components are obtained after the mean is removed. Only the outboard stations ranging from $r / R=0.4$ to 0.51 are considered for the investigation. The effect of blade structural property changes is seen to be significant for both oscillatory bending and torsion moments. The 3 per-revolution signal dominant in the measured flap bending moment is correctly predicted with the present measured blade properties, even though the peak-to-peak response is substantially underestimated. Predictions of both Yeo and Johnson with DEI properties show opposite phases in the first quadrant of the rotor disk, against the measured flap bending signal. The out of phase problem in this region appears greatly fixed with the present predictions. The chord bending shows less satisfactory correlation but the general trend with 4 per-revolution behavior is captured with slight phase shifts. The present torsion moment predictions show good correlation with the measured data. Both magnitudes and phases of the measured torsion moment are predicted reasonably, while Yeo and Johnson with the original DEl properties shows relatively poor correlation. It is remarkable that this poor correlation seems fixed reasonably by simply adjusting the blade structural properties in the tuning process to match the measured frequencies of the rotor. As observed in the flap bending moment correlation, however, this kind of ad hoc process can not guarantee the accuracy of the solution.

\subsection{Airloads}

The correlation of section airloads for the cases with $\mathrm{HHC}$ pitch inputs is made between the present predictions and the measured data. Figure 15 shows the $\mathrm{MN}$ case results at far outboard radial stations $r / R=0.87$ and 0.97 , respectively. The 3 perrevolution behavior due to the $\mathrm{HHC}$ input is clearly captured with correct phase angles. However, the negative loadings apparent near the azimuth angles of $60 \mathrm{deg}$ and $180 \mathrm{deg}$ in the measured airloads are not predicted well with the present comprehensive analysis. This limited prediction becomes more pronounced near the tip of the blade ( $r / R=0.97)$. At this specific location, the mean value is significantly over-estimated compared with the measured data. This limitation could be overcame by introducing the modern CFD (Computational Fluid Dynamics) flow analysis.

Figure 16 shows the comparison of airloads at the two outboard radial stations for the MV case. The correlation is less satisfactory than the $\mathrm{BL}$ and $\mathrm{MN}$ cases. The general loading pattern in the measured signals is predicted but with significantly-reduced peak-to-peak magnitudes. The oscillatory BVI peaks in both advancing and retreating sides are fairly predicted with the present comprehensive analysis. More refined representation of the aerodynamic model appears required for more close correlation.

\subsection{Structural loads}

For HART I test, a total of 32 strain gauges distributed along the span of the instrumented blade (blade 1) were used to measure the structural moments. These measured data were decomposed into harmonic components up to the eighth harmonics using the Fourier series and given in the test report [17]. Figure 17 shows the comparison results of the flap bending moments obtained at $r / R$ $=0.15$ for the three test cases of HART I, between the present predictions and the measured signals. Among the 13 strain gauge locations for the measured flap bending moments, the inboard station located at $15 \%$ radial station is selected since this region is crucial in providing the necessary flexibility of the hingeless configuration such as HART I rotor. Overall, fair to good correlation is obtained. The peak-to-peak values are reasonably predicted with some phase shifts for BL and MN cases, whereas in the MV case, the phase is correctly predicted with substantially decreased peak-to-peak magnitudes. This is related with a smaller prediction in the peak magnitudes of the section normal forces, as observed in Figure 16, which affects on the blade response about 90 deg later.

In Figure 18, the predicted chord bending moments at $15 \%$ radial station for the three test cases of HART I rotor are compared against the measured data. A good correlation in terms of magnitudes and phases of the signal is obtained for all cases, even though some higher harmonic contents observed in the measured data are less dominant in the predicted results. Finally, the comparison is made for the oscillatory torsion moments obtained at $40 \%$ radial station. The correlation is excellent for $\mathrm{BL}$ uncontrolled case and reasonable for cases with $\mathrm{HHC}$ pitch inputs. Again, the phase response is correctly predicted, but the magnitudes are generally under-estimated for both MN and MV cases. Overall, the structural loads correlation is good for the chordwise bending and torsion moments, and fair for the flap bending moments.

\section{CONCLUSIONS}

In this work, the elastic properties of HART I blades were measured using well-established test techniques and a comprehensive rotor analysis was carried out to identify the impact of using the newly measured properties in correlation with wind tunnel test data. The following conclusions were drawn from the current investigation:

1) The blade properties were measured using several test techniques. Both flap and chordwise bending stiffnesses in the blade uniform sections were measured using the 3-point bending method, while the flap bending in the inboard root section and the torsion stiffness in the blade uniform section were measured using the mirror method. The inertia properties were measured using the trifilar pendulum technique. 
2) The measured blade properties were compared with the estimated values provided by DEI who designed and built the HART I blades. It was found that the deviation was up to $30 \%$ for the flap bending over the blade root sections and $14.3 \%$ for the chord bending over the blade uniform section. As compared with the DEl estimations, the measured elastic axis was located more toward the leading edge by about $3 \%$ chord length and the center of gravity was located more aft toward the trailing edge by about $2 \%$ chord length.

3) The comprehensive dynamics analysis demonstrated that a significant improvement in airloads and structural loads predictions was obtained using the measured blade property set. Specifically, an anomaly seen in the waveform of the earlier predictions on the flap bending moments was correctly predicted with the present approach. However, the negative loading patterns found for the $\mathrm{MN}$ and MV cases were not captured correctly in the present comprehensive analysis. More refined representation of the aerodynamic models such as CFD was recommended to capture the detailed flow physics of the problem.

\section{ACKNOWLEDGEMENT}

This research was supported by Leading Foreign Research Institute Recruitment Program through the National Research Foundation of Korea (NRF) funded by the Ministry of Education, Science and Technology (MEST) (K2060100001). The authors appreciate Berend van der Wall of DLR for providing HART I blades, William Warmbrodt of NASA Ames and Thomas Maier of AFDD (US Army Aeroflightdynamics Directorate) for valuable comments on this work. The authors would like to acknowledge the support of HART I partners - DLR, DNW, NASA, ONERA, and AFDD. The authors acknowledge Benton Lau who began the blade property measurement but passed away before the completion of the task.

\section{REFERENCES}

[1] Yu, Y. H., Gmelin, B., Heller, H., Philippe, J. J., Mercker, E., and Preisser, J. S., HHC Aeroacoustics Rotor Test at the DNW - The Joint German/ French/US HART Project, 20th European Rotorcraft Forum, Amsterdam, Netherlands, 1994.

[2] Johnson, W., Milestones in Rotorcraft Aeromechanics, NASA TP-2011-215971, 2011.

[3] Yu, Y. H., Tung, C., van der Wall, B. G., Pausder, H. J., Burley, C., Brooks, T., Beaumier, P., Delriuex, Y., Mercker, E. and Pengel, K., The HARTII Test: Rotor Wakes and Aeroacoustics with HigherHarmonic Pitch Control (HHC) Inputs - The Joint German/French/Dutch/US Project, AHS Int. $58^{\text {th }}$ Annual Forum \& Technology Display, Montreal, Canada. June 11-13, 2002.
[4] van der Wall, B. G., A Comprehensive RotaryWing Database for Code Validation: The HART II International Workshop, Aeronautical Journal, Vol. 115, No. 1163, 2011, pp. 91-102.

[5] Tung, C., Gallman, J. M., Kube, R., Wagner, W., van der Wall, B., Brooks, T. F., Burley, C. L., Boyd, Jr., D. D., Rahier, G., and Beaumier, P., Prediction and Measurement of Blade-Vortex Interaction Loading, CEAS/AIAA Aeroacoustics Conference, Munich, Germany, June 12-15, 1995.

[6] Beaumier, P. and Spiegel, P., Validation of ONERA Aeroacoustic Prediction Methods for BladeVortex Interaction using HART Tests Results, AHS Int. 51 ${ }^{\text {st }}$ Annual Forum \& Technology Display, Fort Worth, TX, May 9-11, 1995.

[7] Brooks, T., Boyd, D. Jr., Burley, C., and Jolly Jr., $\mathrm{J}$, Aeroacoustics Codes for Rotor Harmonic and BVI Noise CAMRAD.Mod1/ HIRES, 2nd CEAS/AIAA Aeroacoustics Conference, State College, PA, May 1996.

[8] Lim, J. W., Tung, C., and Yu, Y. H., Prediction of Blade-Vortex Interaction Airloads with HigherHarmonic Pitch Controls Using the 2GCHAS Comprehensive Code, Journal of Pressure Vessel Technology, Vol. 123, No. 4, Nov. 2001, pp. 469474.

[9] Lim, J. W., Yu, Y. H., and Johnson, W., Calculation of Rotor Blade-Vortex Interaction Airloads Using a Multiple-Trailer Free-Wake Model, Journal of Aircraft, Vol. 40, No. 6, Nov.-Dec. 2003, pp. 1123-1130.

[10] Yeo, H., and Johnson, W., Assessment of Comprehensive Analysis Calculation of Airloads on Helicopter Rotors, Journal of Aircraft, Vol. 42, No. 5, 2005, pp. 1218-1228.

[11] Yeo, H., and Johnson, W., Prediction of Rotor Structural Loads with Comprehensive Analysis, Journal of the American Helicopter Society, Vol. 53, No. 2, 2008, pp. 193-209.

[12] Shekoski, T., TRAM L. H. Blade Qualification and Accepatance Data Report \& Instrumentation and Calibration Report (ATI Document No. 167), Advanced Technologies, Inc., July 1996.

[13] Ogorkiewicz, R. M., and Mucc, P. E. R., Testing of Fibre-Plastics Composites in Three-Point Bending, Composites, Vol. 2, No. 3, 1971, pp. 139145.

[14] Hughes, G. W., The Trifilar Pendulum and Its Application to the Experimental Determination of Moments of Inertia, ASME Paper 57-SA-51, 1957.

[15] Sharpe, D. L., An Experimental Investigation of the Flap-Lag-Torsion Aeroelastic Stability of a SmallScale Hingeless Helicopter Rotor in Hover, NASA TP-2546 (AVSCOM TR 85-A-9), Jan. 1986.

[16] Johnson, W., CAMRAD II: Comprehensive Analytical Model of Rotorcraft Aerodynamics and Dynamics, Johnson Aeronautics, Palo Alto, CA, 1992.

[17] Splettstoesser, W. R., Kube, R., Seelhorst, U., Wagner, W., Boutier, A., Micheli, F., Mercker, E. and Pengel, K., Higher Harmonic Control Aeroacoustic 
Rotor Test (HART) - Test Documentation and Representative Results, Institute Report IB 12995/28, German Aerospace Center (DLR), Braunschweig, Germany, Dec. 1995.

[18] Gere, J. M., and Timoshenko, S. P., Mechanics of Materials, $6^{\text {th }}$ ed., BCT Learning, Belmont, 2004.

[19] van der Wall, B. G., Lim, J. W., Smith, M. J., Jung, S. N., Bailly, J., Baeder, J. D., and Boyd, Jr., D. D., Assessment of Comprehensive Code Prediction State-of-the Art Using the HART International Workshop Data, AHS Int. $68^{\text {th }}$ Annual Forum \& Technology Display, Fort Worth, TX, May 1-3, 2012.

[20] Park, J. S., You, Y. H., Sa, J. H., Park, S. H., and Jung, S. N., Extensive Validation of CFD/CSD Aeroelastic Simulations for a Helicopter in Descending Flight, AHS Int. $68^{\text {th }}$ Annual Forum \& Technology Display, Fort Worth, TX, May 1-3, 2012.

Table 1. Comparison of blade structural properties of HART I uniform section.

\begin{tabular}{ccc}
\hline Property & DEI & Measured \\
\hline Flap bending & $190.1 \mathrm{~N}-\mathrm{m}^{2}$ & $185.6 \mathrm{~N}-\mathrm{m}^{2}$ \\
Lag bending & $4706 \mathrm{~N}-\mathrm{m}^{2}$ & $4032 \mathrm{~N}-\mathrm{m}^{2}$ \\
Torsion stiffness & $134 \mathrm{~N}-\mathrm{m}^{2}$ & $128.5 \mathrm{~N}-\mathrm{m}^{2}$ \\
Center of gravity & $0.245 \mathrm{c}$ & $0.265 \mathrm{c}$ \\
Elastic axis & $0.20 \mathrm{c}$ & $0.169 \mathrm{c}$ \\
Polar MOI at CG & $0.000623 \mathrm{~kg}-\mathrm{m}$ & $0.569 \mathrm{~kg}-\mathrm{m}$ \\
\hline
\end{tabular}

Table 2. HART I trim targets.

\begin{tabular}{cccc}
\hline Property & BL & MN & MV \\
\hline Thrust, N & 3100 & 2964 & 3159 \\
Hub roll, N-m & 11.2 & -15.9 & 21.3 \\
Hub pitch, N-m & -20.0 & -83.6 & 29.1 \\
\hline
\end{tabular}

Table 3. Comparison of rotor trim control angles.

\begin{tabular}{|c|c|c|}
\hline Property & Measured & Present \\
\hline \multicolumn{3}{|c|}{$\mathrm{BL}$} \\
\hline Collective, deg & 3.18 & 3.83 \\
\hline Lateral cyclic, deg & 1.97 & 1.23 \\
\hline Longitudinal cyclic, deg & -1.05 & -1.19 \\
\hline \multicolumn{3}{|c|}{$\begin{array}{ll} & \mathrm{MN} \\
\end{array}$} \\
\hline Collective, deg & 3.15 & 3.78 \\
\hline Lateral cyclic, deg & 1.07 & 1.30 \\
\hline Longitudinal cyclic, deg & -2.04 & -1.26 \\
\hline \multicolumn{3}{|c|}{ MV } \\
\hline Collective, deg & 3.16 & 3.85 \\
\hline Lateral cyclic, deg & 1.11 & 1.28 \\
\hline Longitudinal cyclic, deg & -1.92 & -1.04 \\
\hline
\end{tabular}

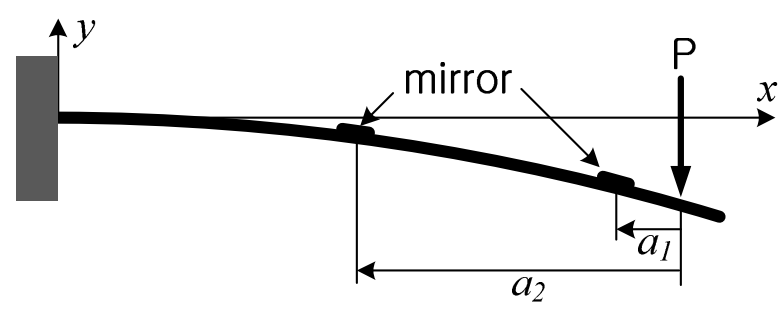

Fig. 1 The mirror method for a blade in bending.

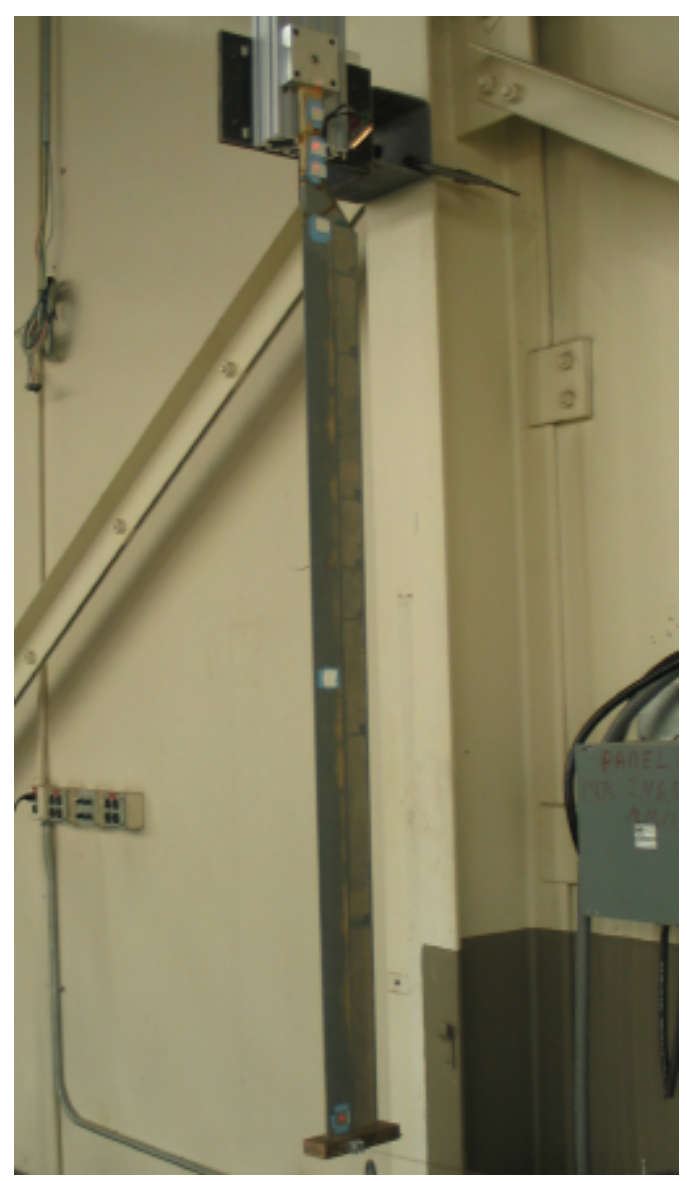

Fig. 2 Measurement of the flap bending for HART I blade with the mirror method.

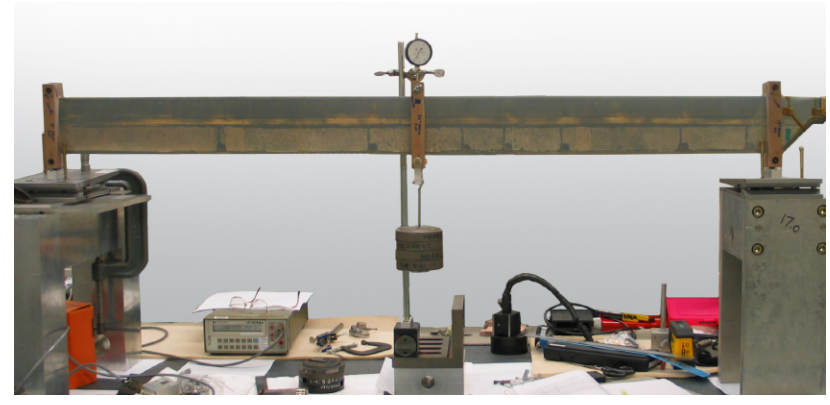

Fig. 3 Measurement of the chordwise bending using a 3-point bending method. 


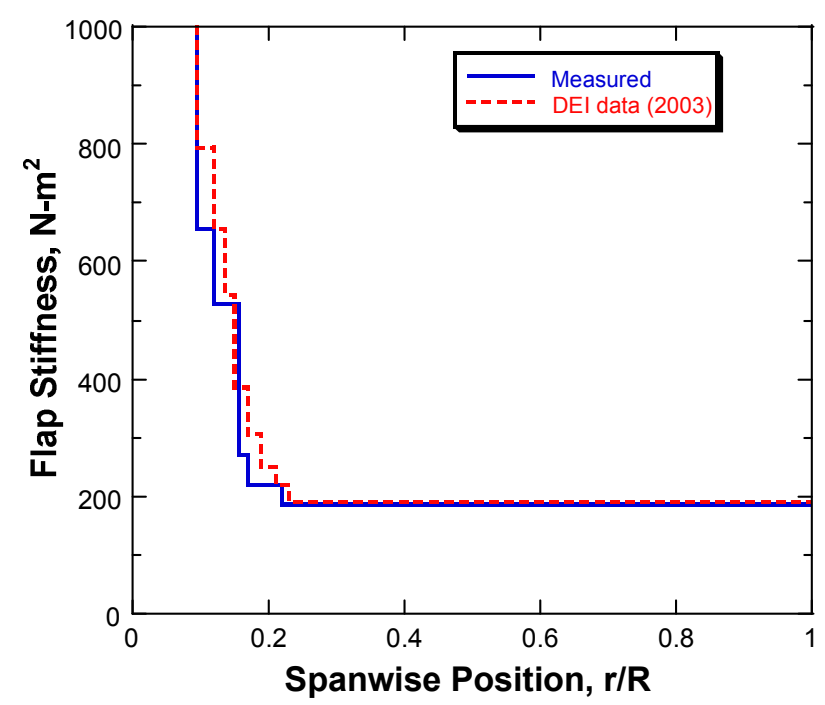

(a) Flap bending

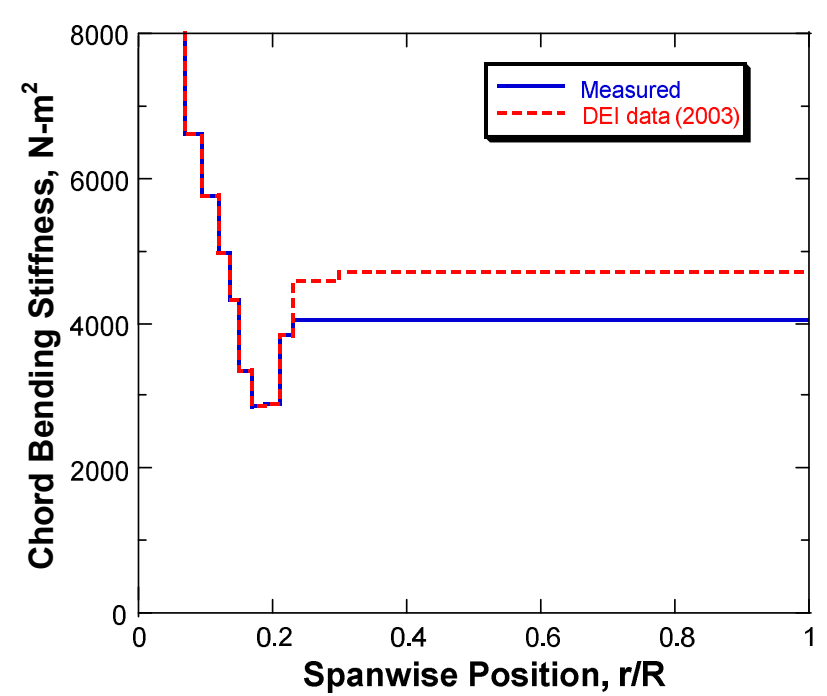

(b) Lead-lag bending

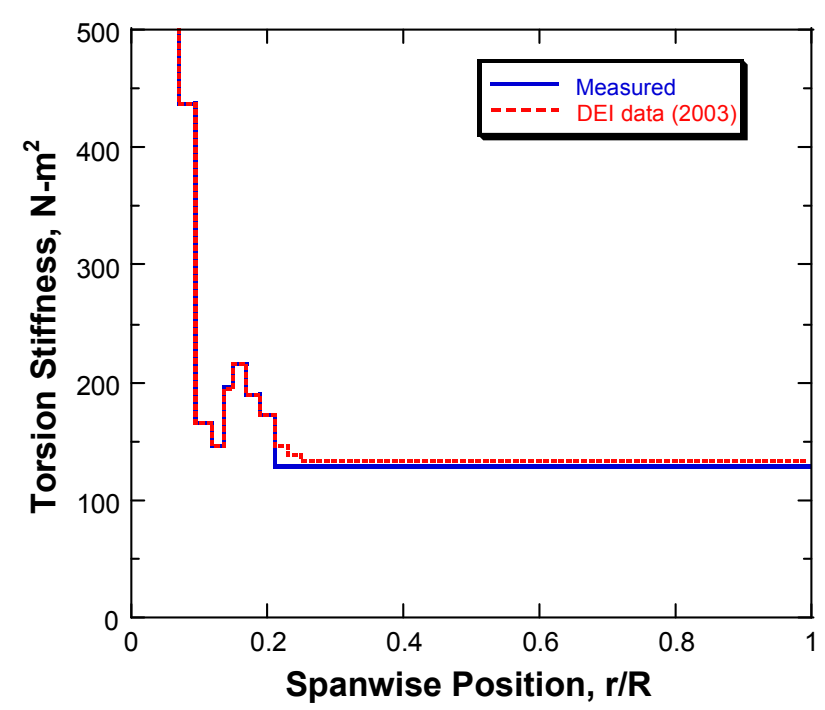

(c) Torsion

Fig. 4 Comparison of section stiffnesses between measurements and DEI data.

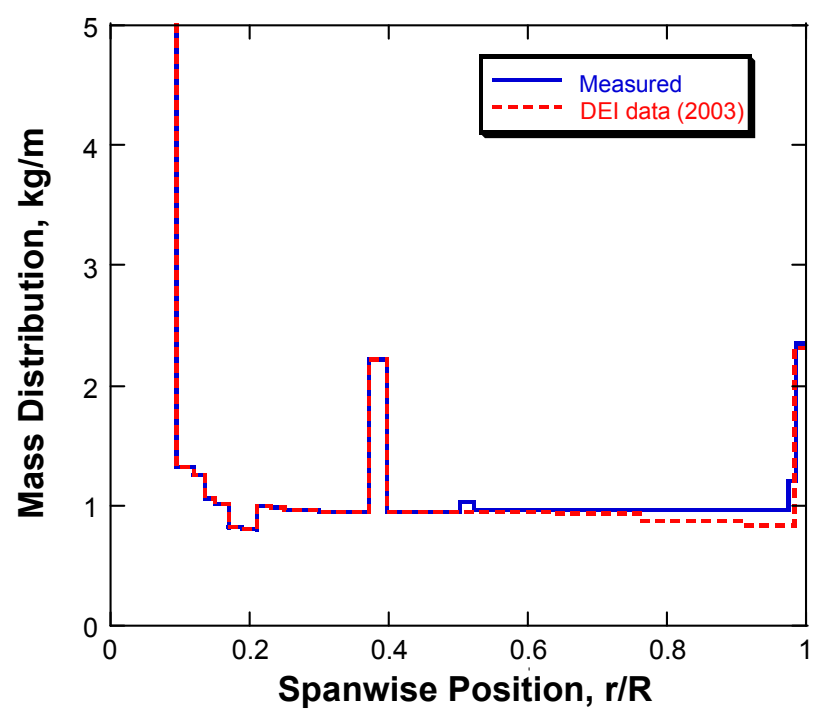

Fig. 5 Comparison of the mass distribution between measurements and DEI data.

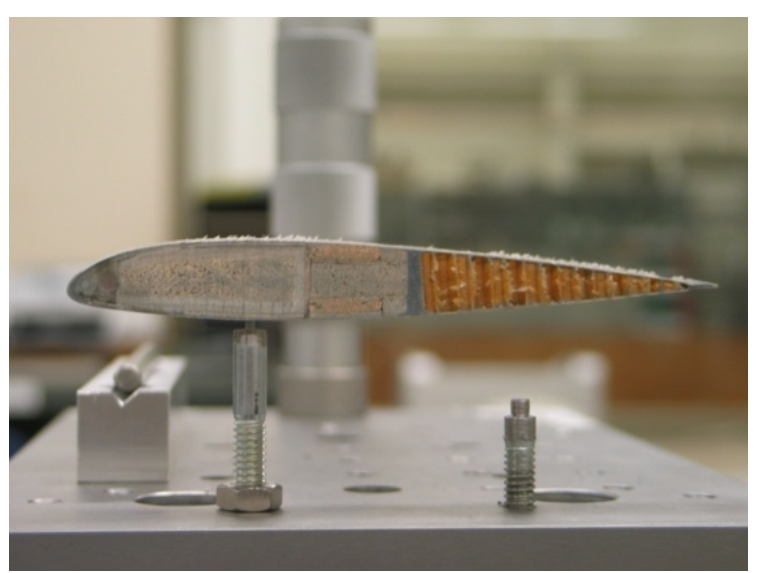

Fig. 6 Measurement of the chordwise center of gravity.

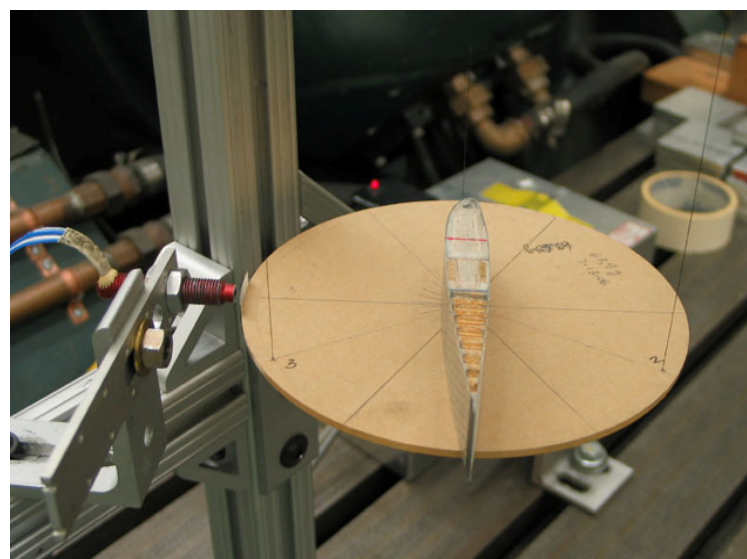

Fig. 7 Measurement of the polar mass moment of inertia using a trifilar pendulum. 


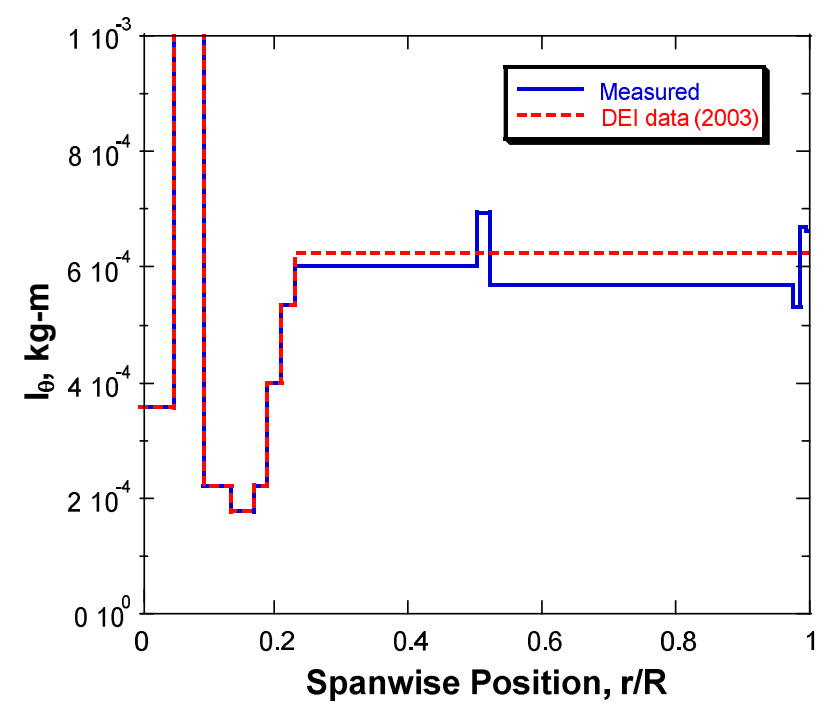

Fig. 8 Comparison of polar mass moment of inertia between measurements and DEI data.

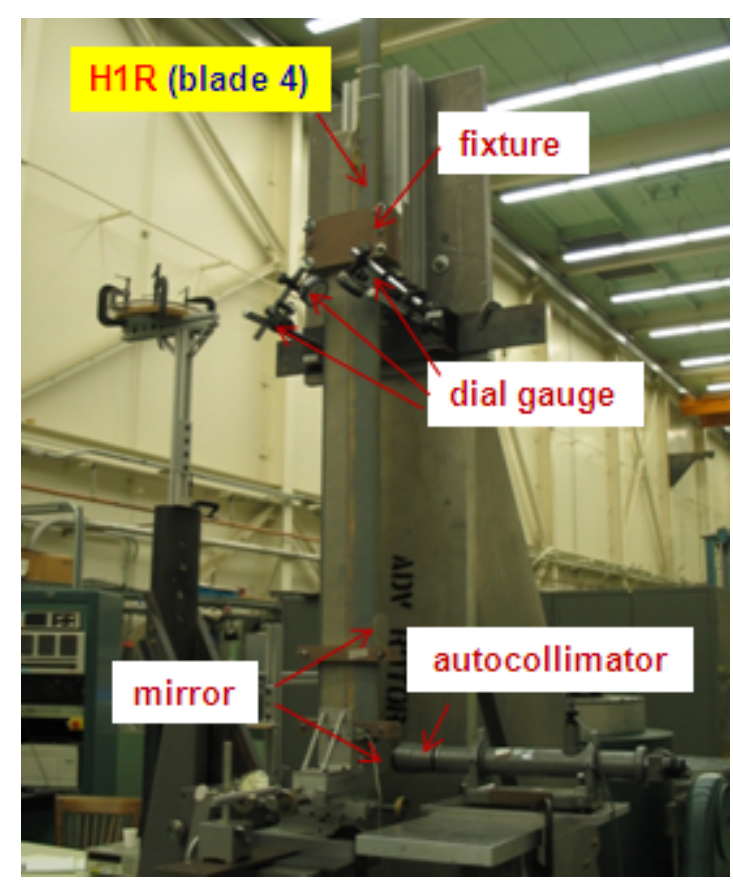

Fig. 9 Measurement setup for the determination of elastic axis.

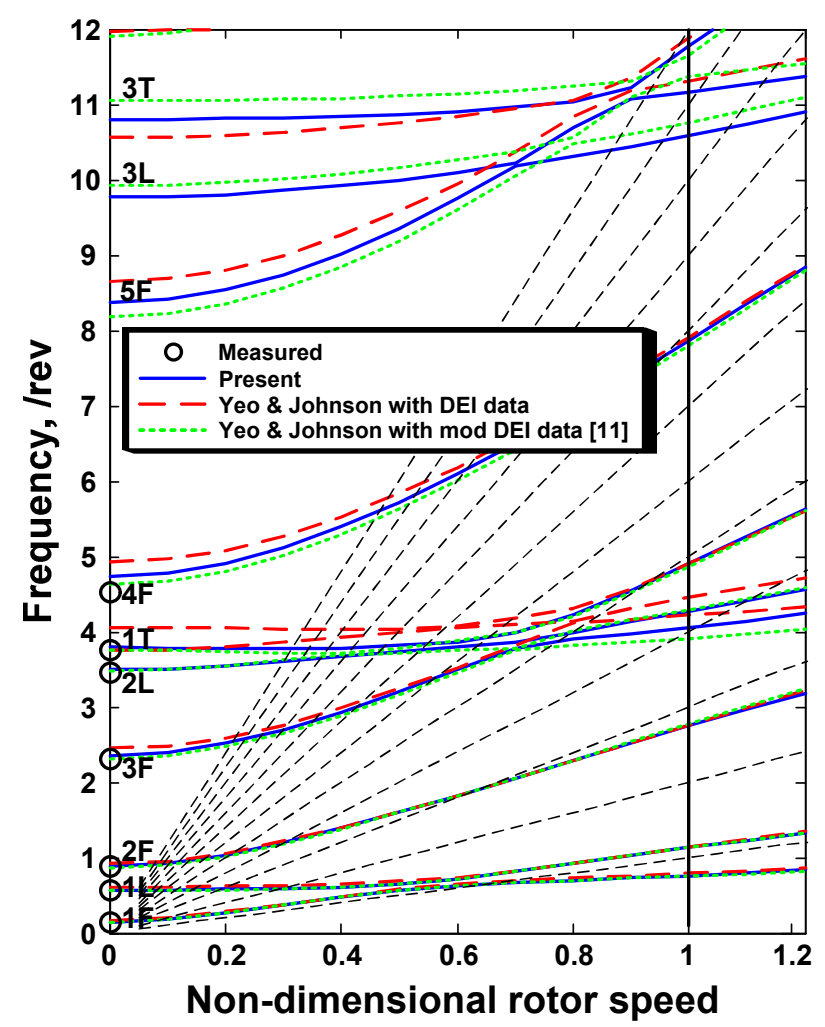

Fig. 10 Comparison of rotor natural frequencies.

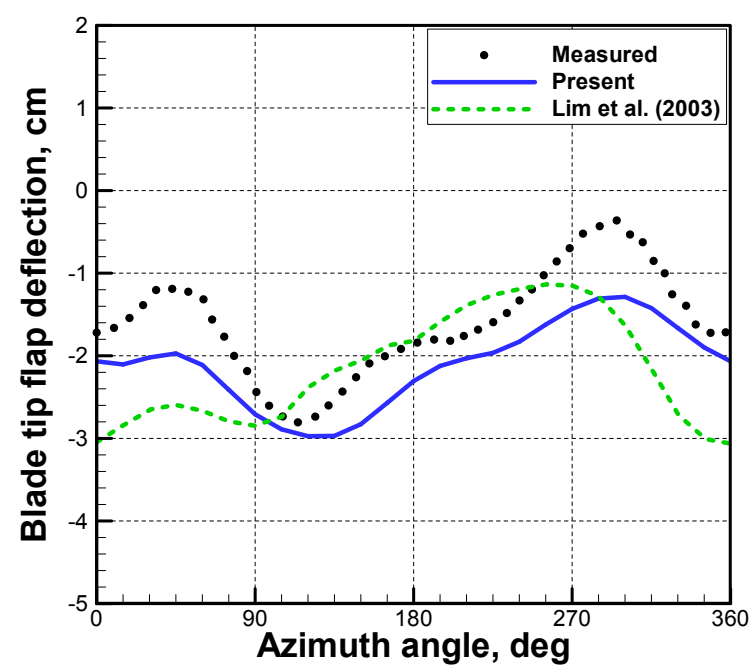

(a) $\mathrm{BL}$ 


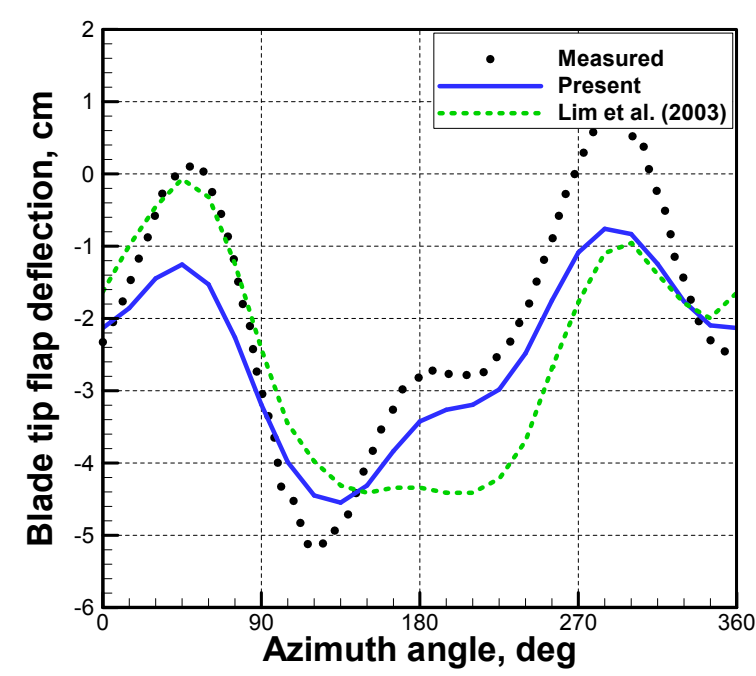

(b) $\mathrm{MN}$

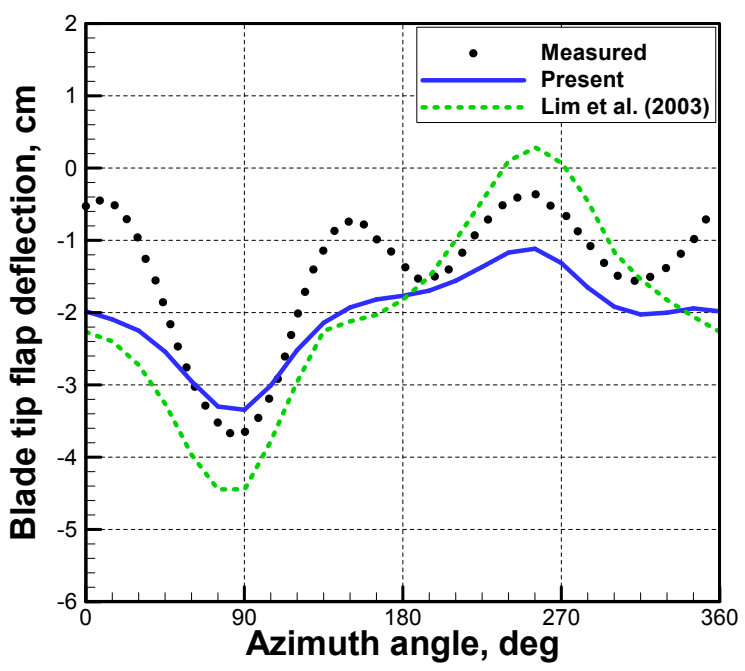

(c) MV

Fig. 11 Validation of predicted flap deflections at the blade tip.

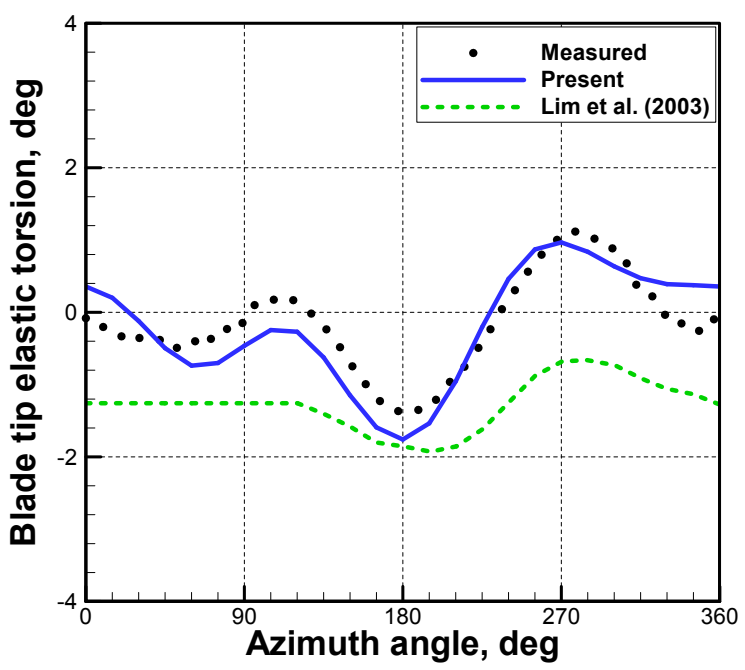

(a) $\mathrm{BL}$

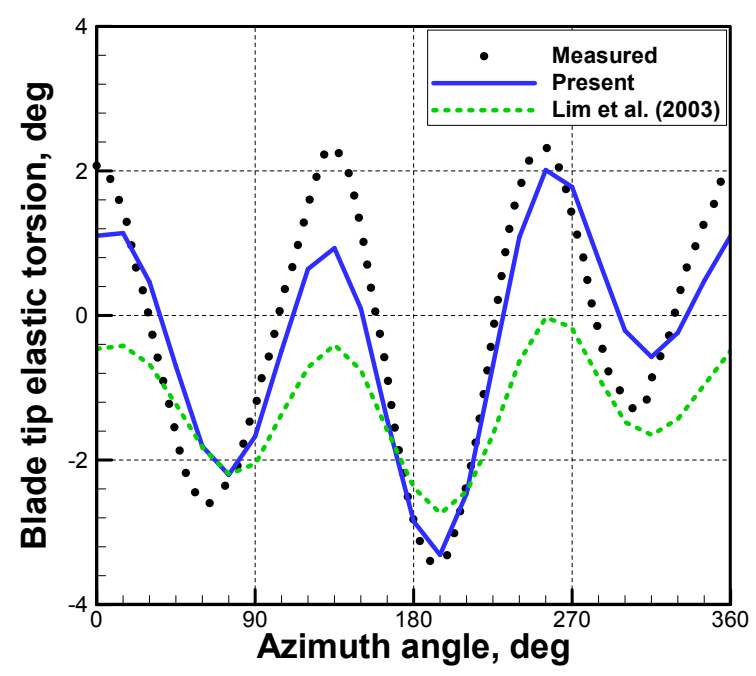

(b) $\mathrm{MN}$

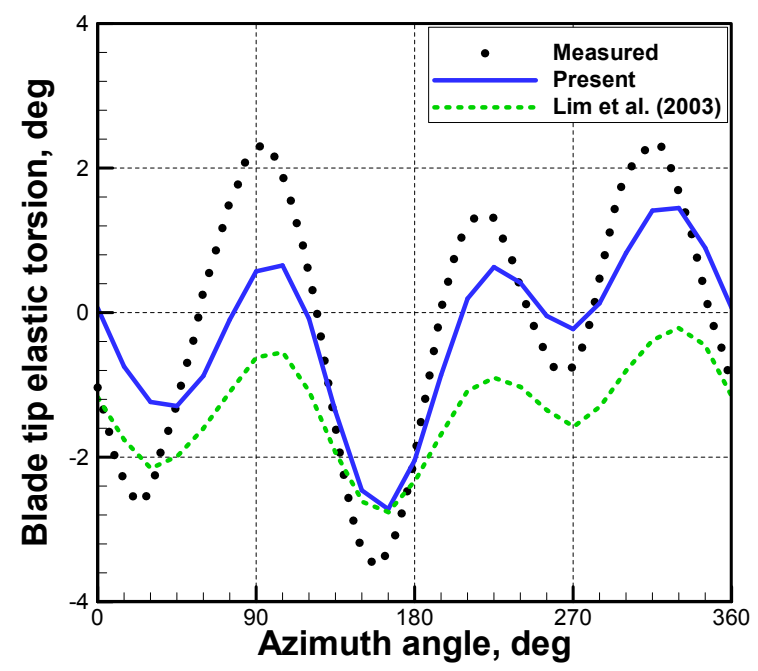

(c) MV

Fig. 12 Validation of predicted twist deformation at the blade tip.

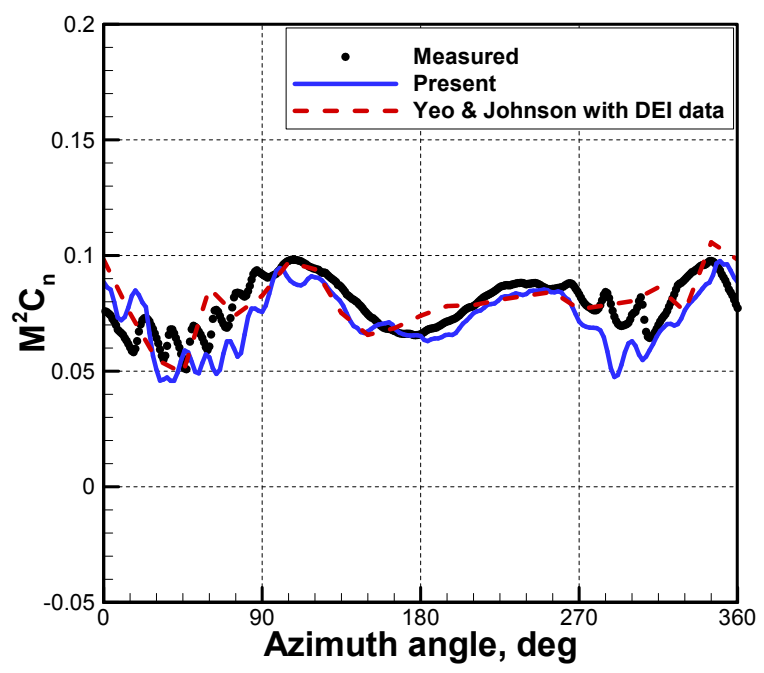

(a) $r / R=0.75$ 


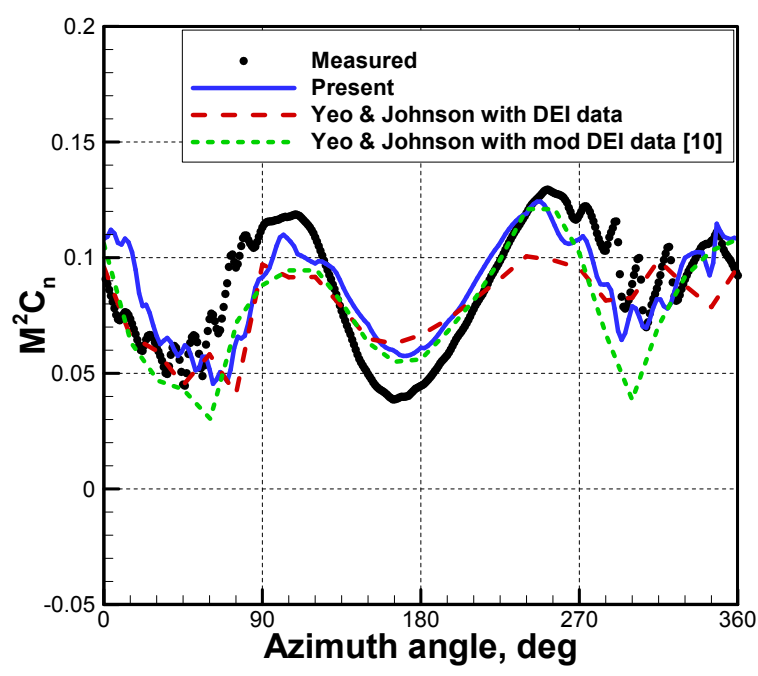

(b) $r / R=0.87$

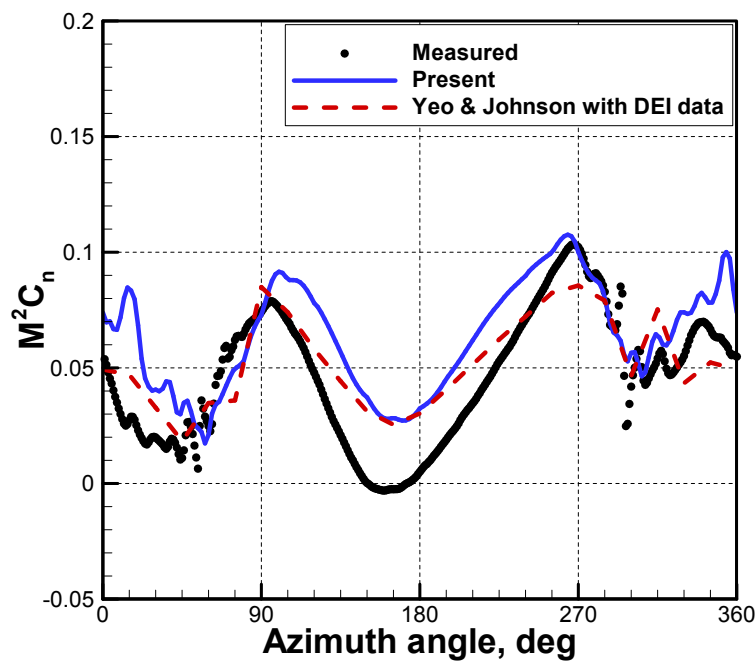

(c) $\mathbf{r} / \mathbf{R}=\mathbf{0 . 9 7}$

Fig. 13 Effect of blade elastic properties on airloads for BL case.

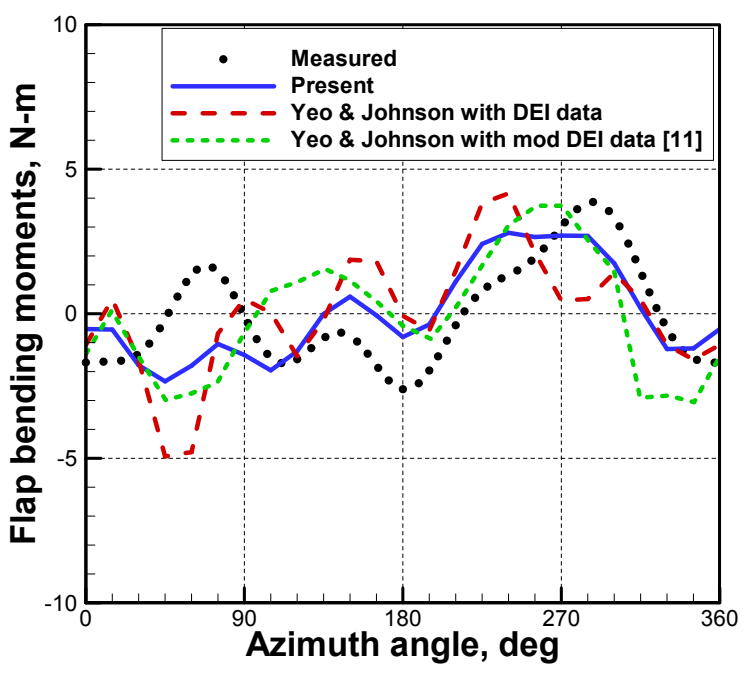

(a) Flap bending $(r / R=0.45)$

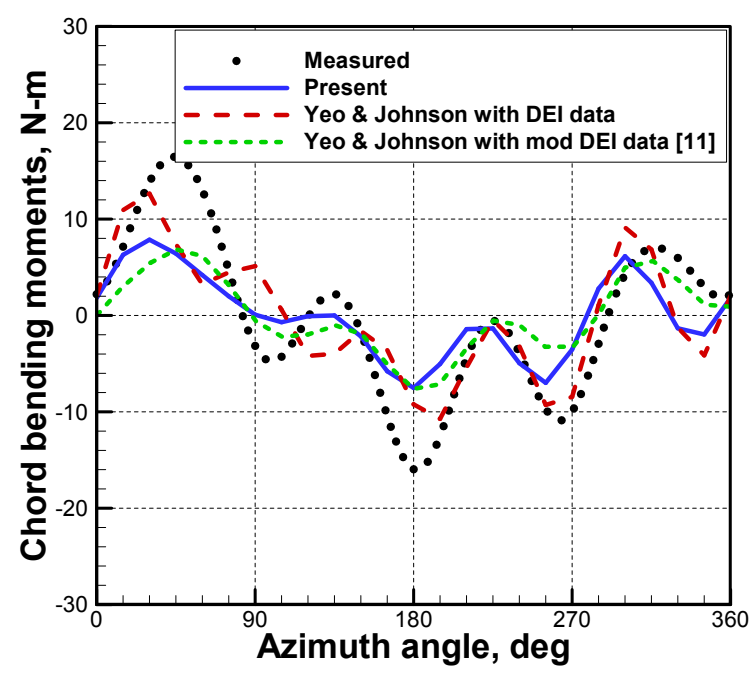

(b) Chord bending $(r / R=0.51)$

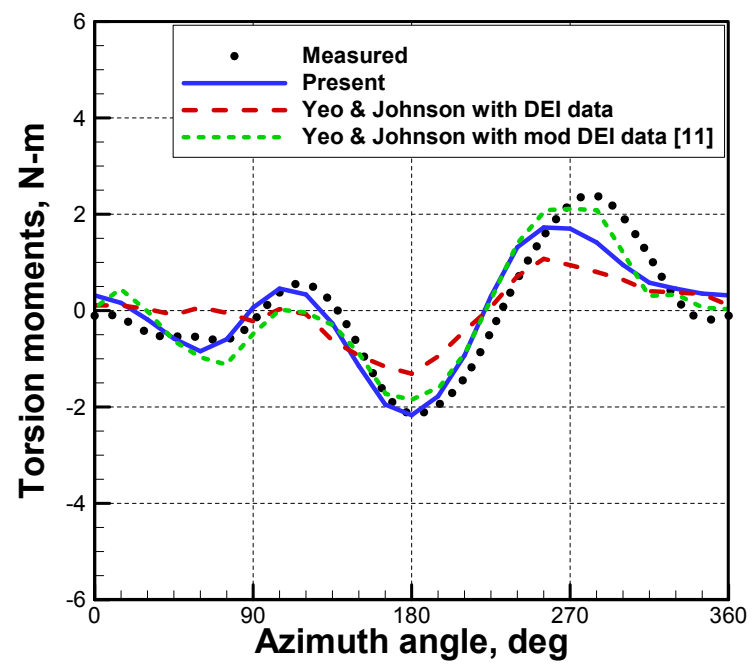

(c) Torsion moment $(r / R=0.40)$

Fig. 14 Effect of blade elastic properties on oscillatory structural loads for BL case.

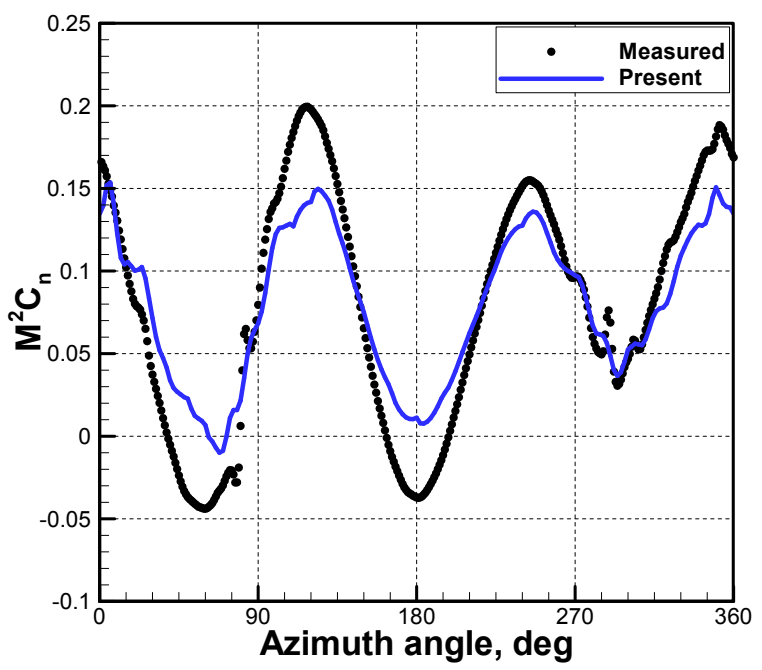

(a) $r / R=0.87$ 


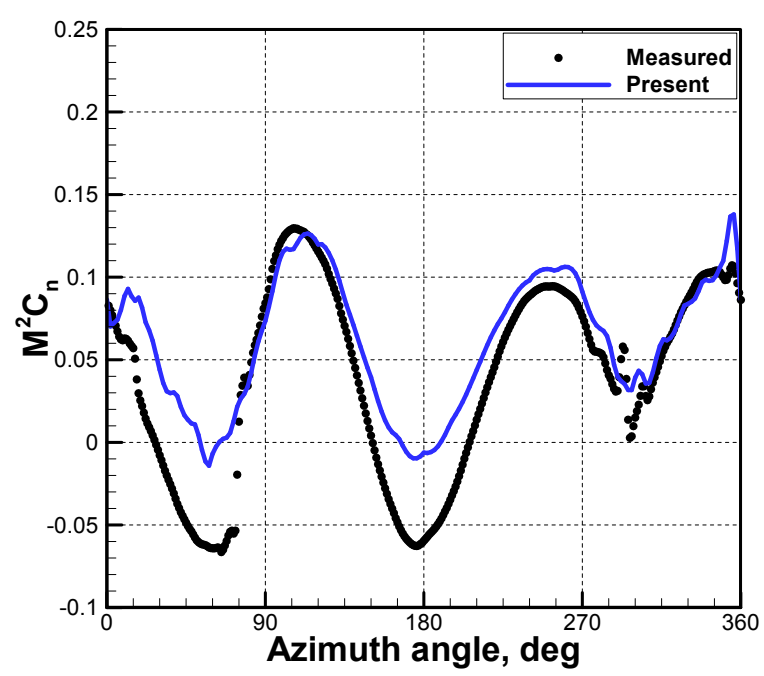

(b) $\mathbf{r} / \mathbf{R}=\mathbf{0 . 9 7}$

Fig. 15 Validation of predicted airloads for MN case.

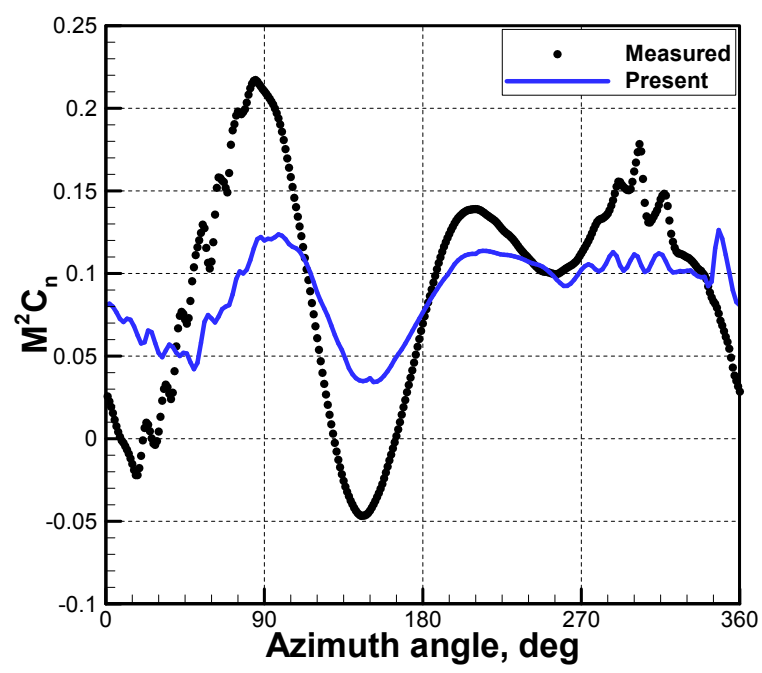

(a) $\mathbf{r} / \mathbf{R}=\mathbf{0 . 8 7}$

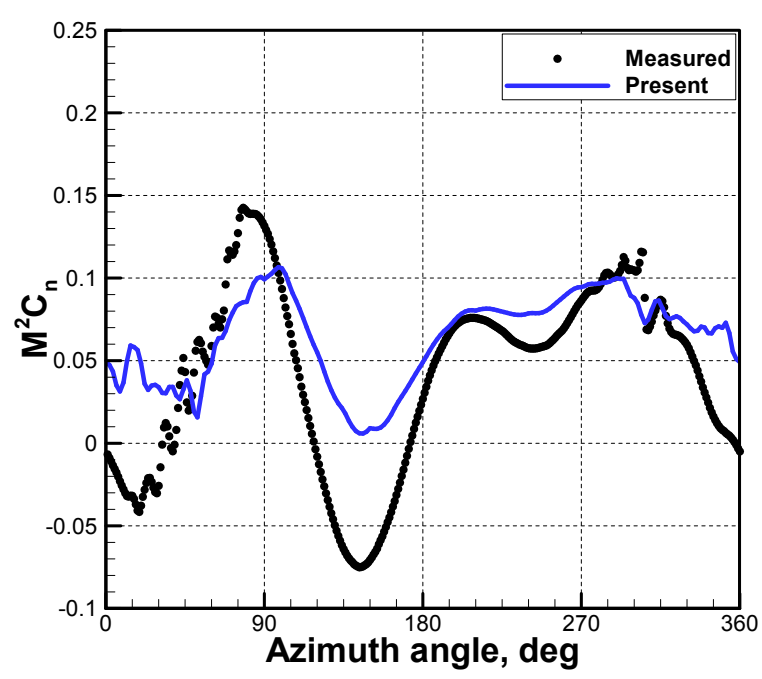

(b) $\mathbf{r} / \mathbf{R}=\mathbf{0 . 9 7}$

Fig. 16 Validation of predicted airloads for MV case.

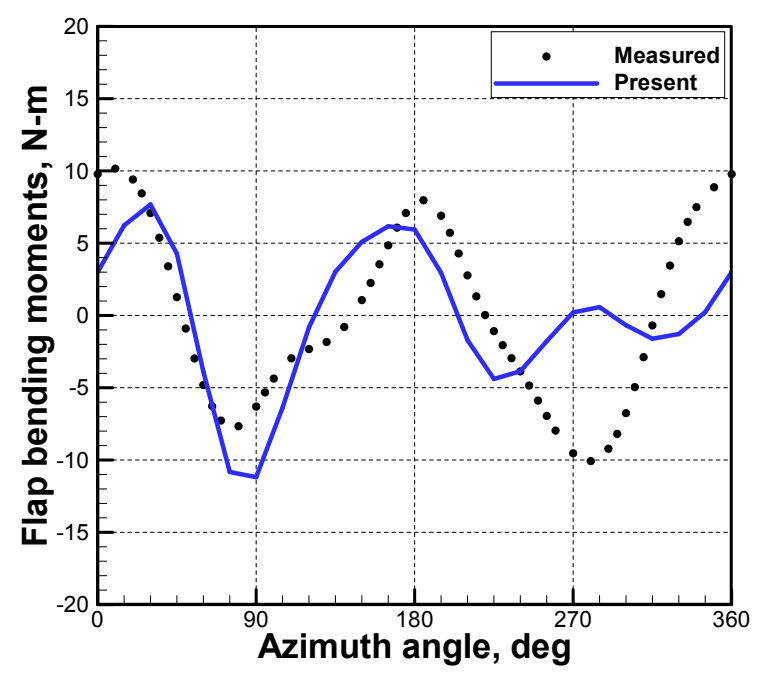

(a) BL

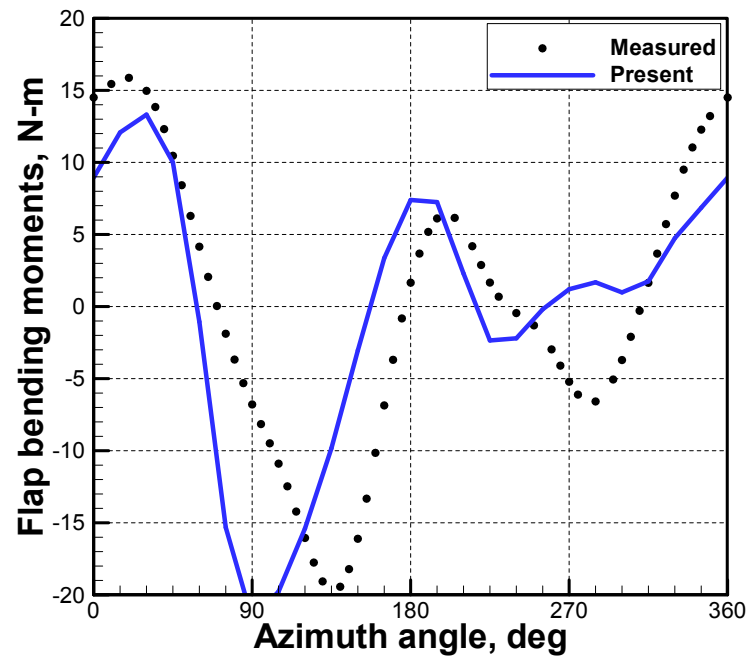

(b) $\mathrm{MN}$

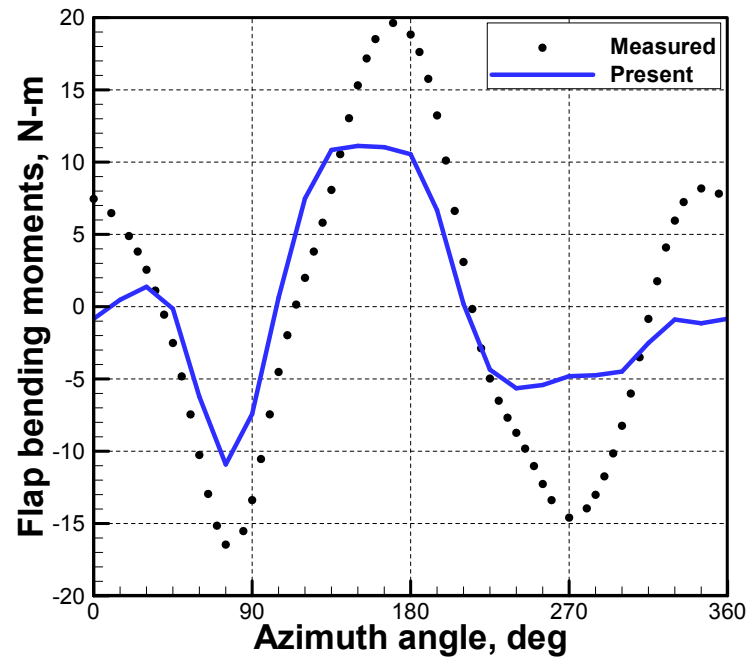

(c) MV

Fig. 17 Validation of predicted oscillatory flap bending moments of HART I blade at $\mathbf{r} / \mathbf{R}=\mathbf{0 . 1 5}$. 


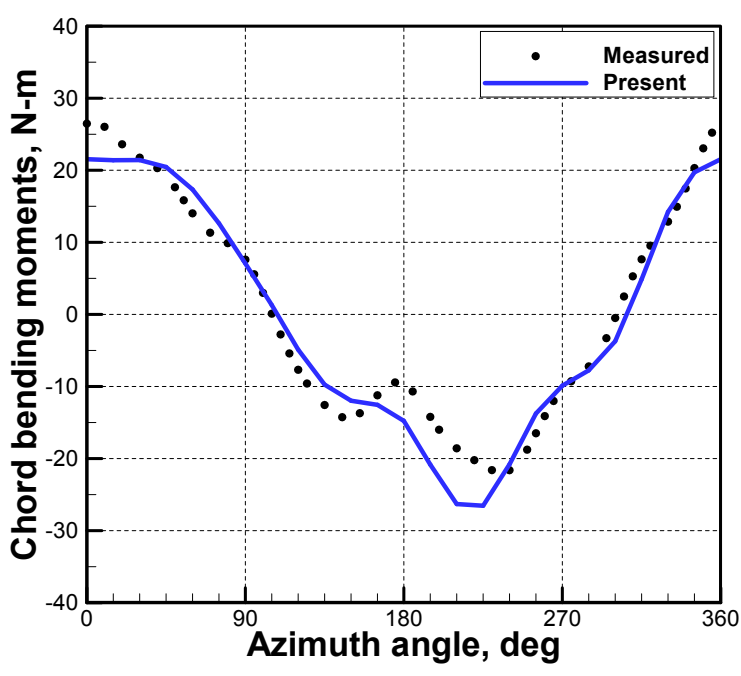

(a) BL

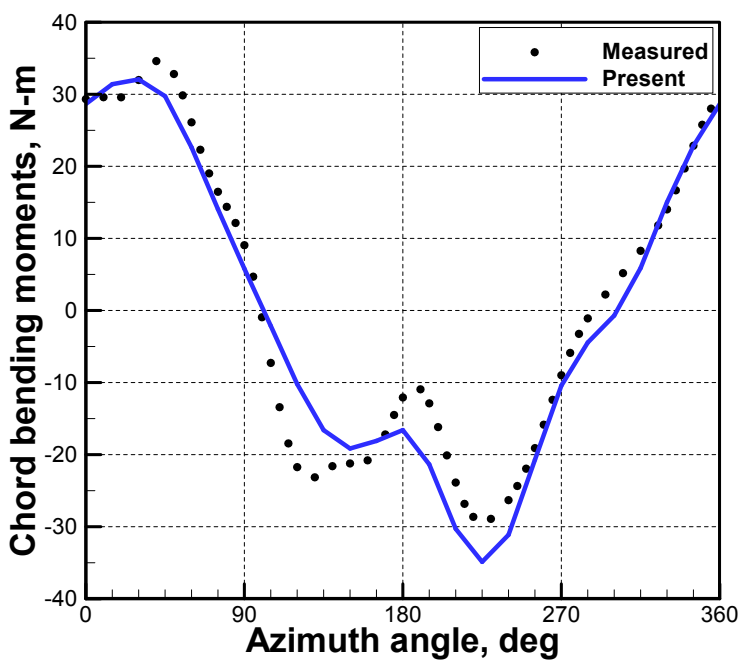

(b) $\mathrm{MN}$

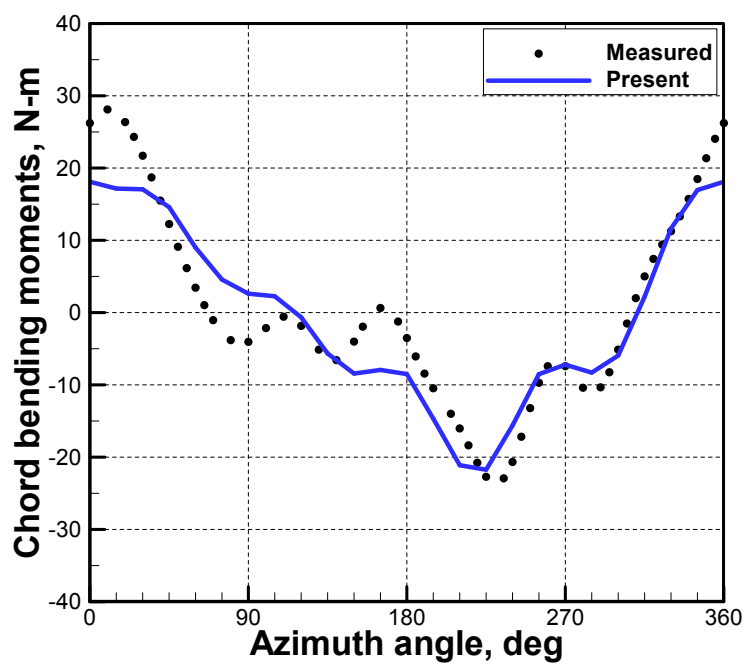

(c) $\mathrm{MV}$

Fig. 18 Validation of predicted oscillatory chord bending moments of HART I blade at $r / R=0.15$.

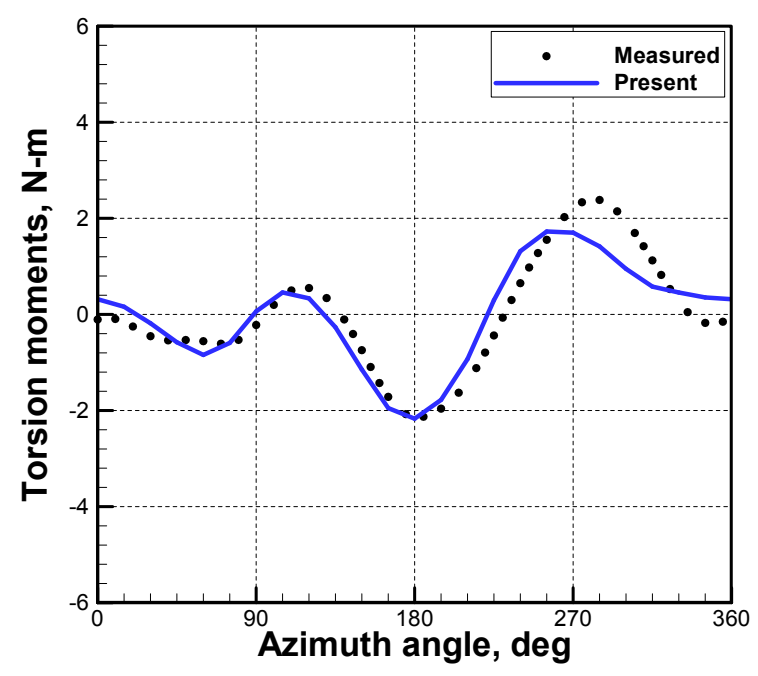

(a) BL

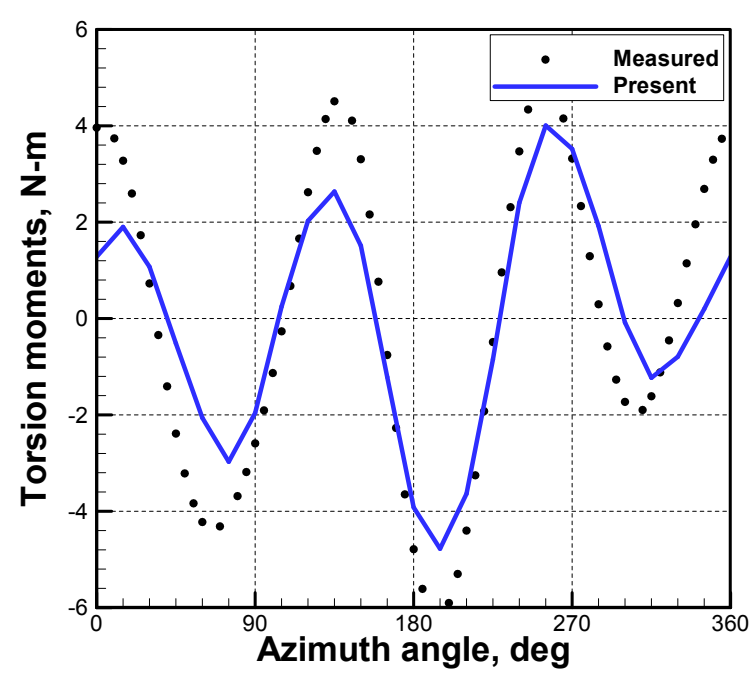

(b) MN

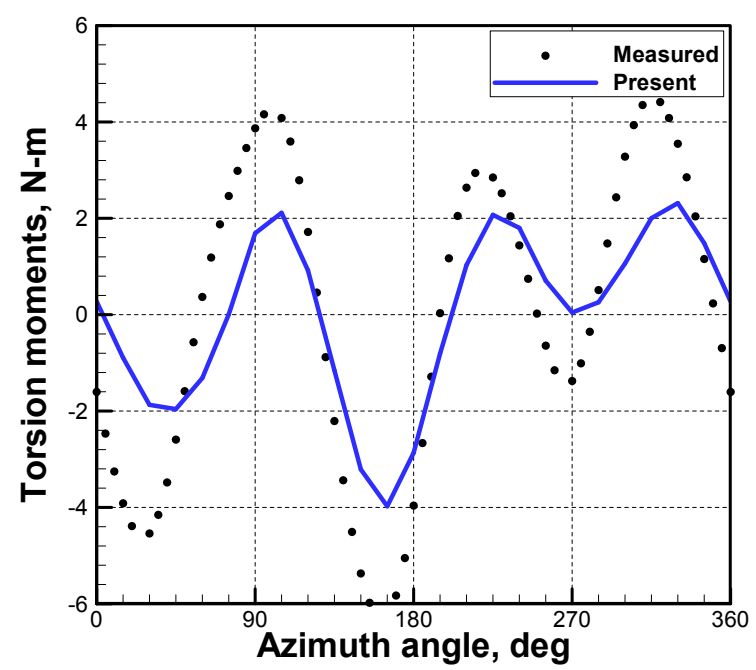

(c) $\mathrm{MV}$

Fig. 19 Validation of predicted oscillatory torsion moments of HART I blade at $r / R=0.4$. 